\title{
Histoculture drug response assay predicts the postoperative prognosis of patients with esophageal cancer
}

\author{
YOSHIHISA FUJITA, MASAKO HIRAMATSU, MASARU KAWAI, HARUTO NISHIMURA, \\ AKIKO MIYAMOTO and NOBUHIKO TANIGAWA \\ Department of General and Gastroenterological Surgery, Osaka Medical College, \\ 2-7 Daigaku-machi, Takatsuki-city, Osaka 569-8686, Japan
}

Received September 9, 2008; Accepted October 27, 2008

DOI: 10.3892/or_00000250

\begin{abstract}
Predicting response to chemotherapy would provide patients suffering from malignant tumor with not only more favorable outcomes, but also reduction of adverse events, and would enable chemotherapy tailored to individual patients. The purpose of this retrospective study was to evaluate the utility of histoculture drug response assay (HDRA) with the MTT endpoint. Subjects comprised 53 consecutive patients diagnosed with esophageal cancer, with 15 patients receiving preoperative chemoradiotherapy (CRT) followed by surgery (CRT group; $\mathrm{n}=15$ ) and 38 patients undergoing surgery (surgery group; $\mathrm{n}=38$ ), including 17 patients with histological lymph node metastasis who received postoperative chemotherapy. Tumor samples obtained from patients were used for HDRA with MTT endpoint and correlations of sensitivity from HDRA with MTT endpoint to clinical response to preoperative CRT, accuracy of in vitro sensitivity test, and clinical outcomes based on HDRA sensitivity were analyzed. HDRA was able to evaluate 379 of 424 assays (89.3\%). In the CRT group, no significant correlation was confirmed between efficacy rate of 5-fluorouracil or cisplatin and histological findings in resected specimens after CRT. Efficacy rates of several anticancer agents using HDRA in the surgery group were observed in the range of $0.0-44.8 \%$. On examination of clinical outcomes in the surgery group, in which patients with stage III received adjuvant chemotherapy, chemosensitivity-negative patients tended to display worse prognosis than chemosensitivity-positive patients. HDRA with MTT endpoint probably predicts the postoperative prognosis of patients with esophageal cancer.
\end{abstract}

Correspondence to: Dr Nobuhiko Tanigawa, Department of General and Gastroenterological Surgery, Osaka Medical College, 2-7 Daigaku-machi, Takatsuki-city, Osaka 569-8686, Japan E-mail:sur001@poh.osaka-med.ac.jp

Key words: chemosensitivity, histoculture drug response assay, esophageal cancer

\section{Introduction}

Esophageal cancer, mainly comprising adenocarcinoma arising from the distal esophagus and esophagealgastric junction, is one of the most common and fatal malignancies in the world (1). In contrast, the most common histological type of esophageal cancer in Japan remains squamous cell carcinoma, with $>9,000$ patients dying of esophageal cancer every year and esophageal cancer representing the sixth and the thirteenth leading cause of death in Japanese males and females, respectively (2). Esophageal cancer, in either histological type, displays highly aggressive behavior with rapid direct invasion of adjacent organs such as the aorta, trachea and left main bronchus and a tendency toward early metastasis to lymph nodes $(3,4)$. To improve outcomes for therapy of esophageal cancer, extended radical esophagectomy with 3-field lymph node dissection has been performed mainly in Japan since 1980 , along with pre- and/or post-operative multimodal therapy including systemic chemotherapy, radiotherapy and concurrent chemoradiotherapy (CRT) since 1990 (5). However, 5 -year survival rate is $5-20 \%$ for stage III and stage IV patients undergoing curative resection in Japan (6), and postoperative recurrence rate remains high, at $\sim 43.3-54.4 \%$ $(7,8)$. Decreasing the rate of postoperative recurrence for esophageal cancer and optimizing treatments for recurrent esophageal cancer are thus crucial.

The two-drug combination of cisplatin (CDDP) and continuous infusion of 5-fluorouracil $(5-\mathrm{Fu})$ is the most common standard regimen for the treatment of esophageal cancer (9). In a phase-III randomized study on esophageal cancer [Japan Clinical Oncology Group (JCOG) study JCOG9204], postoperative adjuvant chemotherapy with CDDP and 5-Fu was better able to prevent relapse in patients with esophageal cancer than surgery alone (10). However, possible adverse events such as hematological toxicity, renal dysfunction and others following repeated administration of CDDP and 5-Fu still affect patients with less sensitivity to CDDP and 5-Fu. A variety of anticancer chemotherapeutic regimens using single or combined new active agents such as docetaxel (TXT) $(11,12)$, paclitaxel (TXL) (13), irinotecan (CPT-11) (14) and gemcitabine $(15,16)$ have recently been developed for the treatment of esophageal cancer and are expected to improve outcomes for esophageal cancer patients. 
Prediction of response to chemotherapy would provide patients suffering from malignant tumor with not only more favorable outcomes, but also reduction in the frequency and severity of adverse events caused by anticancer agents and, in the future, will enable chemotherapy tailored to the individual patient. Although recent advances in molecular cellular biology have led to a better understanding of the mechanisms by which tolerance to anticancer agents is acquired and the molecular factors involved in chemosensitivity (17-20), little information has been available regarding the prediction of chemosensitivity in clinical applications due to reliability, convenience and cost disadvantages.

Hoffman et al (21) introduced a developmental methodology called collagen sponge-gel-supported histoculture, which permits diverse human tumors obtained directly from surgery to grow at high frequency in vitro for long periods of time. The in vivo properties of cultured tumor cells in this method reflect the properties in vitro, including threedimensional growth and maintenance of tissue organization and structure. Given the characteristics of this method, they applied the technique to chemosensitivity assay and established the histoculture drug response assay (HDRA) (22). More recently, Furukawa et al (23) reported that HDRA with the 3-(4,5-dimethylthiazol-2-yl)-2,5-diphenyl-2H tetrazolium bromide (MTT) endpoint is clinically useful for chemosensitivity assay in the field of gastrointestinal surgery field. Although several studies have investigated the efficacy of HDRA with MTT endpoint in a variety of cancers (24-28), little work has been done to elucidate the clinical utility of HDRA with MTT endpoint for esophageal cancer (29).

We performed HDRA with MTT endpoint for patients with esophageal cancer who have undergone operation in our institution since 2004. The present retro-spective study aimed to elucidate the propriety and utility of HDRA with MTT endpoint in esophageal cancer patients.

\section{Patients and methods}

Patient selection and study design. Subjects comprised 53 consecutive patients with esophageal cancer who had undergone surgery between 2004 and 2007 in the Department of General and Gastroenterological Surgery at Osaka Medical College. Fifteen patients received treatment with preoperative CRT followed by surgery (CRT group; $n=15$ ), and 38 patients were treated surgically (surgery group; $n=38$ ), including 17 patients with histological lymph node metastasis who received postoperative chemotherapy. Fresh tumor tissues were collected from the primary lesion during esophagectomy after obtaining written informed consent for use of HDRA with MTT endpoint. The experimental protocol for HDRA with MTT endpoint was approved by the Institutional Review Board of Osaka Medical College. Pathological staging of the esophageal cancer was determined according to Guidelines for the clinical and pathological studies on carcinoma of the esophagus established by the Japanese Society for Esophageal Diseases (30). We analyzed the results obtained from HDRA with MTT endpoint as compared histological response observed in the resected specimens from 18 patients in the CRT group, and clarified the efficacy rate of several anticancer drugs made from HDRA. Finally, we divided patients in the surgery group into four groups according to whether postoperative chemotherapy was administered and whether sensitivity to CDDP or 5-Fu was identified, then assessed the clinical outcomes for patients in each group.

Treatment plan. Preoperative CRT involved low-dose CDDP/5-Fu with concurrent irradiation, comprising 1-h infusion of CDDP at $5 \mathrm{mg}$ /day just before irradiation and continuous 24-h infusion of 5-Fu at $1000 \mathrm{mg} /$ body/every other day, with concurrent irradiation of 2 Gy/day. These drugs were only administered on the days when irradiation was performed. This regimen was performed for 3 weeks and total dose of irradiation was $30 \mathrm{~Gy}$, with esophagectomy performed after a 3-week interval. In the surgery group, adjuvant chemotherapy comprised CDDP at $40 \mathrm{mg} / \mathrm{m}^{2}$ over a 1 -h infusion on day 1 and $5-\mathrm{Fu}$ at $400 \mathrm{mg} / \mathrm{m}^{2}$ as continuous infusion for $24 \mathrm{~h}$ on days 1-5, with standard techniques for hydration and alkalization during hospitalization given to patients with lymph node metastasis. This treatment was repeated every 4 weeks for $>2$ cycles. Based on quality of life and patient status, postoperative chemotherapy was continued with CDDP and 5-Fu chemotherapy or oral chemotherapeutic agents such as oral uracil/tegafur or S-1.

Anticancer agents. Anticancer agents used for testing were 5-Fu, CDDP, adriamycin (ADM), mitomycin C (MMC), TXL, TXT, CPT-11 and gemcitabine (GEM). ADM, 5-Fu and MMC were purchased from Kyowa Hakko Kogyo (Tokyo, Japan), CDDP was from Nippon Kayaku (Tokyo, Japan), TXL came from Bristol-Myers Squibb (New York, NY, USA), TXT was from Sanofi-Aventis (Bridgewater, NJ, USA), CPT-11 was from Daiichi Sankyo (Tokyo, Japan) and GEM was purchased from Eli Lilly and Co. (Indianapolis, IN, USA).

HDRA with MTT endpoint. HDRA with MTT endpoint was performed according to the methods described by Furukawa et al (24) with slight modifications. Collagen sponge gels (Gelfoam; Pfizer, New York, NY, USA) were purchased and the cancerous portion of the surgically resected specimen was cut into pieces using scissors and washed in Hanks' solution with $1 \%$ penicillin/streptomycin (Gibco BRL, Grand Island, NY, USA) and $0.5 \%$ fungizon (Gibco BRL), which were then placed on prepared collagen surfaces in 24-well plates. Anticancer agents were dissolved at various concentrations in RPMI-1640 medium containing 20\% fetal bovine serum (Nikken Cell Media, Kyoto, Japan) and added to each well until the solution reached the upper part of the gel. Plates were then incubated for 7 days at $37^{\circ} \mathrm{C}$ in a humidified atmosphere containing $95 \%$ air and $5 \% \mathrm{CO}_{2}$. After histoculture, a collagenase and MTT solution dissolved in phosphatebuffered saline (PBS) was added to each well and incubation was performed for an additional $8 \mathrm{~h}$. After extracting the MTT-formazan product with dimethyl sulfoxide, absorbance of the solution in each well was read at 540 and $630 \mathrm{~nm}$. Absorbance per gram of histocultured tumor specimen, in drug-treated and non-treated control wells, was calculated by averaging at least three wells. The inhibition rate was then determined using the following formula: Inhibition rate $(\%)$ $=(1-$ mean absorbance per gram of tumor specimen in drugtreated wells/mean absorbance per gram of tumor specimen 
in non-treated control wells) $x 100$. The final concentrations of anticancer agents used in this study were $300 \mu \mathrm{g} / \mathrm{ml}$ for 5-FU, $20 \mu \mathrm{g} / \mathrm{ml}$ for CDDP, $15 \mu \mathrm{g} / \mathrm{ml}$ for ADM, $2.0 \mu \mathrm{g} / \mathrm{ml}$ for MMC, $6.7 \mu \mathrm{g} / \mathrm{ml}$ for TXL, $100 \mu \mathrm{g} / \mathrm{ml}$ for TXT, $10.0 \mu \mathrm{g} / \mathrm{ml}$ for CPT-11 and $80 \mu \mathrm{g} / \mathrm{ml}$ for GEM. Cut-off inhibition rates of CDDP, TXL, TXT, CPT-11 and GEM were set at 50\%, while those of 5-FU, ADM and MMC were 60,60 and 70\%, respectively. When inhibition rates exceeded the individual cut-off value, the tumor was considered chemosensitive to the individual agent. The final drug concentrations of 5-Fu, ADM, CDDP, MMC and TXT were determined according to previously reported values $(24,28)$, whereas final concentrations of TXL, CPT-11 and GEM were defined as peak plasma concentration.

Evaluation of preoperative CRT and postoperative chemotherapy. Tumor response to preoperative CRT was histologically evaluated by resected specimens according to histological criteria as follows: Grade 0, neither necrosis nor cellular or structural changes seen throughout the lesion; Grade 1, necrosis or disappearance of tumor observed, but viable tumor cells remain in $>1 / 3$ of the whole lesion; Grade 2, viable tumor cells remain in $<1 / 3$, i.e., necrosis or disappearance of tumor comprising almost the whole lesion; Grade 3, whole lesion is necrotic and/or replaced by fibrosis, with or without granulomatous changes, and no viable tumor cells are observed. Patients with histological response of Grade 2 or 3 were considered as responders to preoperative CRT, whereas patients with pathological response of Grade 0 or 1 were considered as non-responders to preoperative CRT. Statistical analyses were performed using JMP 6.0 software (SAS Institute, NC, USA). Statistical analysis included Student's t-test, Pearson's $\chi^{2}$ test and Fisher's exact test, as appropriate. Overall survival in the surgery group was measured from the date on which esophagectomy was performed to the date of death or last confirmed date of survival. The Kaplan-Meier method was used to estimate overall survival curves after esophagectomy and each group was compared using log-rank testing. Values of $\mathrm{P}<0.05$ were accepted as statistically significant. Final follow-up was performed in February 2008.

\section{Results}

Evaluability of HDRA with MTT endpoint. A total of 53 consecutive patients with esophageal cancer were examined for sensitivity to 8 individual anticancer drugs using HDRA with MTT endpoint. The result showed that $89.3 \%$ of assays (379/424 total assays) were able to be evaluated using HDRA with MTT endpoint.

Confirmation of relationship between sensitivity to CDDP and 5-Fu obtained from HDRA with MTT endpoint and clinical response to preoperative $C R T$. Fifteen patients in the CRT group were divided into two groups according to histological response as determined by histological findings in resected specimens. The responder group comprised 6 patients in the CRT group diagnosed as Grade 2 or 3, whereas the non-responder group comprised 9 patients in the CRT group diagnosed as Grade 0 or 1. Efficacy rate (determined as the
Table I. Correlation between efficacy rate for chemosensitivity and clinical response to preoperative chemoradiotherapy.

\begin{tabular}{llcc}
\hline & \multicolumn{2}{c}{ Efficacy rate $^{\mathrm{a}}$} & \\
\cline { 2 - 3 } $\begin{array}{l}\text { Anticancer } \\
\text { agent }\end{array}$ & $\begin{array}{c}\text { Responder } \\
\text { group }(\mathrm{n}=6)\end{array}$ & $\begin{array}{c}\text { Non-responder } \\
\text { group (n=9) }\end{array}$ & $\mathrm{P}$ \\
\hline $5-\mathrm{Fu}$ & $50.0 \%(3$ of 6$)$ & $36.6 \%(4$ of 9$)$ & 0.8327 \\
CDDP & $66.6 \%(4$ of 6$)$ & $33.3 \%(3$ of 9$)$ & 0.2049 \\
\hline
\end{tabular}

${ }^{a}$ Efficacy rate was calculated as number of chemosensitive cases/ number of evaluated cases.

Table II. Efficacy rate obtained by HDRA with MTT endpoint in the surgery group.

Efficacy rate

Anticancer agent

(HDRA with MTT endpoint)

\begin{tabular}{lc}
\hline 5 -Fu & $23.7 \%(9 / 38)$ \\
CDDP & $40.5 \%(15 / 37)$ \\
ADM & $0.0 \%(0 / 37)$ \\
MMC & $44.8 \%(13 / 29)$ \\
TXL & $18.9 \%(7 / 37)$ \\
TXT & $27.6 \%(8 / 29)$ \\
CPT-11 & $8.6 \%(3 / 35)$ \\
GEM & $13.8 \%(4 / 29)$ \\
\hline
\end{tabular}

number of chemosensitive cases/number of evaluated cases) for $5-\mathrm{Fu}$ was $50 \%$ (3 of 6) in the responder group, compared to $36.6 \%$ (4 of 9) in the non-responder group (Table I). For CDDP, efficacy rate in responder and non-responder groups was $66.6 \%$ (4 of 6) and 33.3\% (3 of 9), respectively (Table I). No significant differences were observed between groups for either agent (5-Fu, $\mathrm{P}=0.8327$; CDDP, $\mathrm{P}=0.2049$ ).

Efficacy rate for anticancer agents in the surgery group measured by HDRA with MTT endpoint. To assess the reliability of sensitivity obtained by HDRA with MTT endpoint, efficacy rates for anticancer agents in the surgery group were measured by in vitro assay. Analysis of efficacy rate for individual agents measured by HDRA with MTT endpoint showed that MMC (44.8\%) and CDDP (40.5\%) had high efficacy rates, 5-Fu (23.7\%), TXT (27.6\%) and TXL (18.9\%) had moderate efficacy rates, and ADM (0.0\%), CPT-11 (8.6\%) and GEM $(13.8 \%)$ had low efficacy rates (Table II).

Retrospective analysis of clinical outcomes for patients in the surgery group. To evaluate clinical outcomes of patients with esophageal cancer, patients in the surgery group were divided into four groups according to whether postoperative chemotherapy was administered and whether the tumor was chemosensitive to CDDP or 5-Fu, and clinical outcomes were assessed in each group. Patients with chemosensitive tumors were divided into two groups: patients given adjuvant 


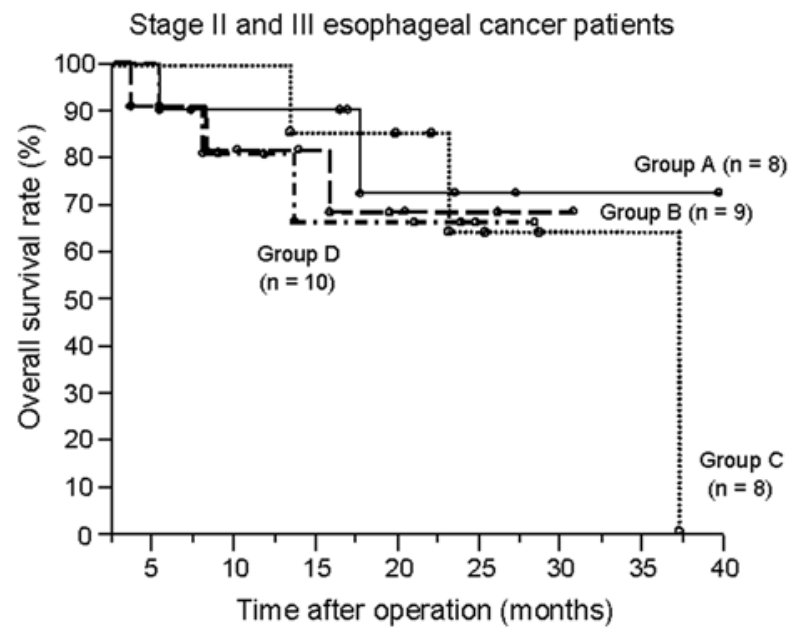

Figure 1. In the surgery group, retrospective analysis of clinical outcomes for patients with stage II $(n=11)$ and III $(n=24)$. Patients in the surgery group were divided into four groups according to whether postoperative chemotherapy was administered and whether the tumor was chemosensitive to CDDP or 5-Fu, and clinical outcomes were assessed in each group. Patients with chemosensitive tumors were divided into two groups: patients given adjuvant chemotherapy (group A); and patients not given adjuvant chemotherapy (group B). Patients without chemosensitivity were divided into another two groups: patients given adjuvant chemotherapy (group C); and patients not given adjuvant chemotherapy (group D). Overall survival curves after operation in these four groups were evaluated according to Kaplan-Meier methods and the results revealed no significant differences between group A ( $n=8)$, group B $(n=9)$, group $C(n=8)$ and group $D(n=10)$.

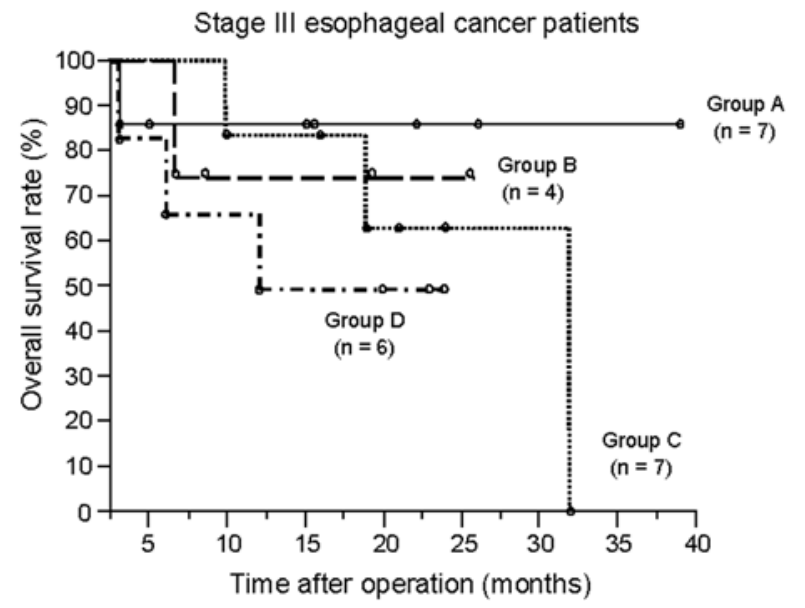

Figure 2. Analysis of overall survival curves after operation restricted to patients with stage III disease $(n=24)$. Patients in group A $(n=7)$ tended to display better prognosis than patients in groups $B(n=4), C(n=7)$ or $D(n=6)$. Comparison of overall survival curves between group A (chemosensitivitypositive) and group $\mathrm{C}$ (chemosensitivity-negative), who were administered adjuvant chemotherapy comprising CDDP and 5-Fu, suggested a lack of significant differences due to censored data $(\mathrm{P}=0.583)$. Although administering adjuvant chemotherapy to patients with stage III disease (group A and $\mathrm{C}$ ) led to a better prognosis, patients in group $\mathrm{C}$ tended to show worse prognosis than patients in group A.

chemotherapy (group A); and patients not given adjuvant chemotherapy (group B). In addition, patients without chemosensitivity were divided into another two groups: patients given adjuvant chemotherapy (group C); and patients not given adjuvant chemotherapy (group D).

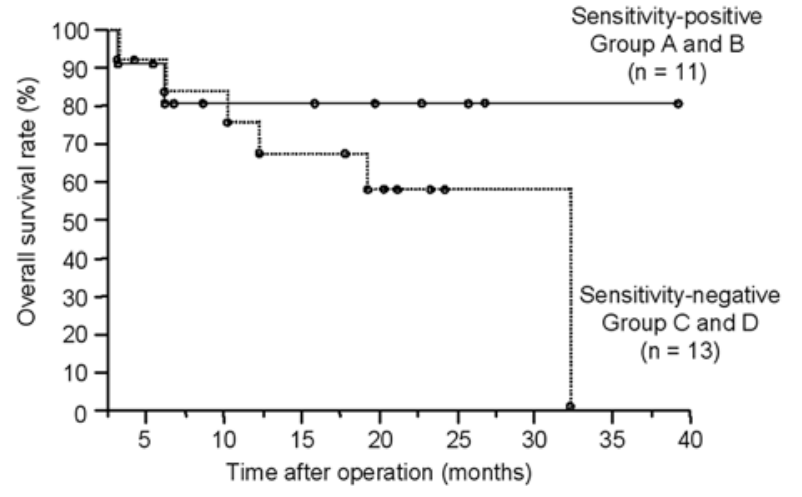

Figure 3. Analysis of overall survival curves after operation restricted to patients with stage III disease $(n=24)$. Patients in chemosensitivity-positive group (group A and B) $(n=11)$ tended to display better prognosis than patients in chemosensitivity-negative group (group C and D) $(n=13)$. Although no significant differences were observed between chemosensitivitypositive group and chemosensitivity-negative group, chemosensitivity obtained from in vitro assay probably promised to predict the postoperative prognosis of esophageal cancer.

Thirty-five patients in the surgery group diagnosed as stage II $(n=11)$ or III $(n=24)$ were eligible for analysis and divided into the four groups mentioned above (Table III). Overall survival curves after operation in these four groups were evaluated according to Kaplan-Meier methods and the results revealed no significant differences between group A $(n=8)$, group $B(n=9)$, group $C(n=8)$ and group D $(n=10)$ (Fig. 1). Conversely, analysis of overall survival curves after operation restricted to patients with stage III disease $(n=24)$ suggested that patients in group $A(n=7)$ tended to display better prognosis than patients in groups B $(n=4), C(n=7)$ or $D(n=6)$ (Fig. 2). In addition, comparison of overall survival curves between group A (chemosensitivity-positive) and group $\mathrm{C}$ (chemosensitivity-negative), who were both administered adjuvant chemotherapy comprising CDDP and $5-\mathrm{Fu}$, suggested a lack of significant differences due to censored data $(\mathrm{P}=0.583)$. Although administering adjuvant chemotherapy to patients with stage III disease (group A and $\mathrm{C}$ ) led to a better prognosis, patients in group $\mathrm{C}$ tended to show worse prognosis than patients in group A.

Finally, we compared the overall survival curve of the patients with stage III between chemosensitivity-positive group (group A and B) and chemosensitivity-negative group (group $\mathrm{C}$ and D). The prognosis of the patients in chemosensitivity-positive tended to be better than the prognosis of those in chemosensitivity-negative group, however, no significant differences between chemosensitivity-positive group and chemosensitivity-negative group was observed. $(\mathrm{P}=0.254)$ (Fig. 3).

\section{Discussion}

The present results show that patients classified as chemosensitivity-negative using in vitro assay displayed worse prognosis than patients classified as chemosensitivitypositive regardress of administering adjuvant chemotherapy. These results indicate that HDRA with MTT endpoint offers promise as a chemosensitivity assay for predicting the efficacy of various anticancer agents in esophageal cancer patients 
Table III. distribution of patients in surgery groups.

\begin{tabular}{lccccc}
\hline $\begin{array}{l}\text { Adjuvant chemotherapy } \\
\text { chemosensitivity }\end{array}$ & \begin{tabular}{c} 
Group A \\
\cline { 5 - 6 }
\end{tabular} & $\begin{array}{c}\text { Given } \\
\text { Positive }\end{array}$ & $\begin{array}{c}\text { Got given } \\
\text { Positive }\end{array}$ & $\begin{array}{c}\text { Given } \\
\text { Negative }\end{array}$ & $\begin{array}{c}\text { Group D } \\
\text { Not given } \\
\text { Negative }\end{array}$ \\
\hline Stage II and III & $\mathrm{n}$ & $\mathrm{n}$ & $\mathrm{n}$ & $\mathrm{n}$ & 10 \\
Stage III & 8 & 9 & 8 & 6 & 35 \\
\hline
\end{tabular}

and chemosensitivity obtained from in vitro assay probably predicts the postoperative prognosis of esophageal cancer.

One of the characteristics of HDRA with MTT endpoint includes high evaluability, which was determined to be $89.3 \%$ in this study. This finding is congruent with other studies concerning a variety of cancers, including head and neck cancer $(28,31)$, gastrointestinal tract cancer $(24,29,32)$, breast cancer $(25,26)$ and urological cancer $(33)$. The reason for this high evaluability is that HDRA with MTT endpoint, as established and modified by Hoffmann et al (34) and Furukawa et al $(24,35)$, enables a native-state histoculture including tissue architecture, tumor-stromal interaction and differentiated functions, a long-term cell culture, and long-term exposure to time-dependent anticancer drugs such as 5-Fu. However, a few studies have mentioned that over-estimation of drug efficacy, specifically false positive, was observed in using MTT endpoint assay for chemosensitivity test $(36,37)$. These findings were observed mainly in the conventional monolayer cell cultures using MTT assay, not in HDRA with MTT endpoint. In the collagen sponge-gel-supported histoculture, the growth rates of the tumors in vitro were about the same as those of the tumors in vivo (38) and the growth rates of stromal cell, including fibroblast, were almost constant and slow in comparison with the monolayer cell cultures in which fibroblast cells grew rapidly in the early phase of cultures (35). Therefore, the influence of normal stromal cells including fibroblast cells on the HDRA with MTT endpoint was negligible. Although some problems, including measurement of cancer tissue of $\sim 1.0 \mathrm{~cm}^{3}$ and contamination of fibroblasts have been mentioned in comparison with collagen gel droplet embedded culture drug sensitivity test (CD-DST) $(39,40)$, HDRA with MTT endpoint offers the advantage of a very high preservation of in vivo tissue architecture observed in vitro compared with CD-DST, which features disaggregated tumor cells and disrupted tumor-stromal architecture $(35,41)$. In addition, MTT end point assay using HDRA is more easily performed and more convenient at research laboratories compared to imaging analysis using CD-DST.

In the present study concerning the CRT group, resected specimens obtained from chemosensitive patients tended to display good response to preoperative CRT. However, no significant correlation between efficacy rate of 5-Fu or CDDP and histological findings in resected specimens after CRT was confirmed. This result was attributed to the fact that subject numbers in the CRT group were too small and patients in the CRT group received treatment not only with chemotherapy comprised CDDP and 5-Fu but also with concurrent irradiation of total $30 \mathrm{~Gy}$. Suda et al (29) reported a significant correlation between the results of assay using HDRA and clinical effects of preoperative chemotherapy, and concluded that HDRA with MTT endpoint should be introduced clinically. Conversely, Kodera et al (32) reported that HDRA with MTT endpoint showed no correlation to clinical findings in a particular subset of patients who underwent preoperative therapy. These differences may be partly explained by tumor specimens obtained after preoperative therapy including CRT and chemotherapy mainly comprising atypical tumor cell clones that have acquired some degree of resistance to anticancer agents administered in preoperative settings and thus would not reflect the original phenotypic characteristics. In a study of correlations between the chemosensitivity of primary breast tumor and lymph node metastases, significant differences in chemosensitivity of each tumor site was observed due to the heterogeneity of metastatic tumors (25).

On examination of the efficacy rate for several anticancer agents in our surgery group, efficacy rate for in vitro assay was observed in the range of $0.0-44.8 \%$. Historical clinical data show response rates to 5-Fu, CDDP, ADM, MMC, TXL, TXT, CPT-11 and GEM of 15\% (4/26) (42), 25\% (6/24) (43), 5\% (1/20) (42), $42 \%$ (10/42) (43), 32\% (16/50) (44), $20 \%(10 / 49)(12), 14 \%(6 / 43)(14)$ and $0.0 \%(0 / 17)(45)$, respectively. Good correlations between efficacy rate for an individual agent using HDRA in vitro assay and clinical response rate to each agent have been reported previously. These findings suggest that chemosensitivity determined from HDRA with MTT endpoint probably reflects chemosensitivity in vivo and would be suitable for clinical applications and are congruent with a number of prior studies reporting HDRA as a useful predictor for response to chemotherapy in various cancer (25-28). These results show that HDRA allows conservation of the original phenotypic characteristics of tumor cells.

Adjuvant chemotherapy based on CDDP and 5-Fu has provided a decrease in recurrence rate after curative resection for esophageal cancer patients with lymph node metastases (JCOG9204) (10). In the present study, retrospective analysis restricted to patients with stage III disease showed that patients in groups A and B, who had undergone adjuvant chemotherapy, tended to show better prognosis than patients in the other two groups. However, examination of 35 patients in the 
surgery group diagnosed with stage II or III disease revealed no significant differences in overall survival between groups, suggesting surgery, not adjuvant chemotherapy, as the main influence on outcomes for patients with stage II disease. As for patients with stage III disease, patients in group $\mathrm{C}$ received adjuvant chemotherapy, but tended to show worse prognosis than patients in group A. More specifically, patients in group C had been diagnosed as chemosensitivity-negative using HDRA with MTT endpoint and would be regarded as resistant to adjuvant chemotherapy based on CDDP and 5-Fu. If resistance to adjuvant chemotherapy is identified in chemosensitivity-negative patients, a second-line chemotherapy should be selected as soon as possible. In addition, as for patients with stage III disease, patients in chemosensitivitypositive group (group A and B) tended to have better prognosis than patients in chemosensitivity-negative group (group C and group D). These results suggested that chemosensitivity obtained from in vitro assay probably predicts the postoperative prognosis of patients with esophageal cancer.

Although a few studies on correlations between patient outcomes and chemosensitivity as determined from HDRA are available $(24,46,47)$, no clinical phase III study of HDRA comparing patient survival for HDRA-based therapy versus physician-selected therapy including surgery alone have yet been performed. The effect of adjuvant chemotherapy in chemosensitive patients has thus not been elucidated. Further examination of HDRA with MTT endpoint including a prospective randomized study is urgently needed.

In conclusion, HDRA with MTT endpoint offers nativestate histoculture and high evaluability, and significantly correlated with response rate as described in previous studies. In addition, patients classified as chemosensitivity-negative using in vitro assay, though they were administered adjuvant chemotherapy, showed worse prognosis than patients classified as chemosensitivity-positive, suggesting that HDRA with MTT endpoint probabl predicts the postoperative prognosis of patients with esophageal cancer.

\section{References}

1. Jemal A, Siegel R, Ward E, et al: Cancer statistics, 2006. CA Cancer J Clin 56: 106-130, 2006.

2. Cancer Statistics in Japan 2007: Foundation for Promotion of Cancer Research. Cancer Statistics in Japan Editorial Board. National Cancer Center, Tokyo, Japan, 2007.

3. Enzinger PC and Mayer RJ: Esophageal cancer. N Engl J Med 349: 2241-2252, 2003.

4. Tew WP, Kelsen DP and Ilson DH: Targeted therapies for esophageal cancer. Oncologist 10: 590-601, 2005.

5. Fujita H, Sueyoshi S, Tanaka T and Shirouzu K: Three-field dissection for squamous cell carcinoma in the thoracic esophagus. Ann Thorac Cardiovasc Surg 8: 328-335, 2002.

6. Registration Committee for Esophageal Cancer: Comprehensive Registry of Esophageal Cancer in Japan $(1998,1999)$ and Longterm Results of Esophagectomy for Esophageal Cancer in Japan (1988-1997). 3rd edition, Japanese Society for Esophageal Dieseases, Tokyo, 2002.

7. Mariette C, Balon JM, Piessen G, Fabre S, Van Seuningen I and Triboulet JP: Pattern of recurrence following complete resection of esophageal carcinoma and factors predictive of recurrent disease. Cancer 97: 1616-1623, 2003.

8. Nakagawa S, Kanda T, Kosugi S, Ohashi M, Suzuki T and Hatakeyama K: Recurrence pattern of squamous cell carcinoma of the thoracic esophagus after extended radical esophagectomy with three-field lymphadenectomy. J Am Coll Surg 198: 205-211, 2004.
9. Iizuka T, Kakegawa T, Ide H, et al: Phase II evaluation of cisplatin and 5-fluorouracil in advanced squamous cell carcinoma of the esophagus: a Japanese Esophageal Oncology Group Trial. Jpn J Clin Oncol 22: 172-176, 1992.

10. Ando N, Iizuka T, Ide H, et al: Surgery plus chemotherapy compared with surgery alone for localized squamous cell carcinoma of the thoracic esophagus: a Japan Clinical Oncology Group Study - JCOG9204. J Clin Oncol 21: 4592-4596, 2003.

11. Govindan R, Read W, Faust J, et al: Phase II study of docetaxel and irinotecan in metastatic or recurrent esophageal cancer: a preliminary report. Oncology 17: 27-31, 2003.

12. Muro K, Hamaguchi T, Ohtsu A, et al: A phase II study of single-agent docetaxel in patients with metastatic esophageal cancer. Ann Oncol 15: 955-959, 2004.

13. Lowy AM, Firdaus I, Roychowdhury D, et al: A phase II study of sequential neoadjuvant gemcitabine and paclitaxel, radiation therapy with cisplatin and 5-fluorouracil and surgery in locally advanced esophageal carcinoma. Am J Clin Oncol 29: 555-561, 2006.

14. Enzinger PC, Kulke MH, Clark JW, et al: A phase II trial of irinotecan in patients with previously untreated advanced esophageal and gastric adenocarcinoma. Dig Dis Sci 50: 2218-2223, 2005.

15. Albertsson M, Johansson B, Friesland S, et al: Phase II studies on docetaxel alone every third week, or weekly in combination with gemcitabine in patients with primary locally advanced, metastatic, or recurrent esophageal cancer. Med Oncol 24: 407-412, 2007.

16. Williamson SK, McCoy SA, Gandara DR, et al: Phase II trial of gemcitabine plus irinotecan in patients with esophageal cancer: a Southwest Oncology Group (SWOG) trial. Am J Clin Oncol 29: 116-122, 2006.

17. Tanaka E, Hashimoto Y, Ito T, et al: The suppression of auroraA/STK15/BTAK expression enhances chemosensitivity to docetaxel in human esophageal squamous cell carcinoma. Clin Cancer Res 13: 1331-1340, 2007.

18. Shimokuni T, Tanimoto K, Hiyama K, et al: Chemosensitivity prediction in esophageal squamous cell carcinoma: novel marker genes and efficacy-prediction formulae using their expression data. Int J Oncol 28: 1153-1162, 2006.

19. Keshelava N, Davicioni E, Wan Z, et al: Histone deacetylase 1 gene expression and sensitization of multidrug-resistant neuroblastoma cell lines to cytotoxic agents by depsipeptide. J Natl Cancer Inst 99: 1107-1119, 2007.

20. Gyorffy B, Surowiak P, Kiesslich O, et al: Gene expression profiling of 30 cancer cell lines predicts resistance towards 11 anticancer drugs at clinically achieved concentrations. Int J Cancer 118: 1699-1712, 2006.

21. Freeman AE and Hoffman RM: In vivo-like growth of human tumors in vitro. Proc Natl Acad Sci USA 83: 2694-2698, 1986.

22. Vescio RA, Redfern CH, Nelson TJ, Ugoretz S, Stern PH and Hoffman RM: In vivo-like drug responses of human tumors growing in three-dimensional gel-supported primary culture. Proc Natl Acad Sci USA 84: 5029-5033, 1987.

23. Furukawa T, Kubota T, Watanabe M, et al: Chemosensitivity testing of clinical gastrointestinal cancers using histoculture and the MTT end-point. Anticancer Res 12: 1377-1382, 1992.

24. Furukawa T, Kubota T and Hoffman RM: Clinical applications of the histoculture drug response assay. Clin Cancer Res 1: 305-311, 1995.

25. Furukawa T, Kubota $\mathrm{T}$, Tanino $\mathrm{H}$, et al: Chemosensitivity of breast cancer lymph node metastasis compared to the primary tumor from individual patients tested in the histoculture drug response assay. Anticancer Res 20: 3657-3658, 2000.

26. Tanino H, Oura S, Hoffman RM, et al: Acquisition of multidrug resistance in recurrent breast cancer demonstrated by the histoculture drug response assay. Anticancer Res 21: 4083-4086, 2001.

27. Yoshimasu T, Oura S, Hirai I, et al: In vitro evaluation of doseresponse curve for paclitaxel in breast cancer. Breast Cancer 14: 401-405, 2007.

28. Ariyoshi Y, Shimahara M and Tanigawa N: Study on chemosensitivity of oral squamous cell carcinomas by histoculture drug response assay. Oral Oncol 39: 701-707, 2003.

29. Suda S, Akiyama S, Sekiguchi H, Kasai Y, Ito K and Nakao A: Evaluation of the histoculture drug response assay as a sensitivity test for anticancer agents. Surg Today 32: 477-481, 2002.

30. Japanese Society for Esophageal Diseases: Guidelines for clinical and pathologic studies on carcinoma of the esophagus, 9th edition: Preface, general principles, part I. Esophagus 1: 61-88, 2004. 
31. Hasegawa Y, Goto M, Hanai N, et al: Evaluation of optimal drug concentration in histoculture drug response assay in association with clinical efficacy for head and neck cancer. Oral Oncol 43: 749-756, 2007.

32. Kodera Y, Ito S, Fujiwara M, et al: In vitro chemosensitivity test to predict chemosensitivity for paclitaxel, using human gastric carcinoma tissues. Int J Clin Oncol 11: 449-453, 2006.

33. Hirano Y, Ushiyama T, Suzuki K and Fujita K: Clinical application of an in vitro chemosensitivity test, the Histoculture Drug Response Assay, to urological cancers: wide distribution of inhibition rates in bladder cancer and renal cell cancer. Urol Res 27: 483-488, 1999.

34. Hoffman RM: Three-dimensional histoculture: origins and applications in cancer research. Cancer Cell 3: 86-92, 1991.

35. Furukawa T, Kubota T, Watanabe M, et al: High in vitroin vivo correlation of drug response using sponge-gel-supported three-dimensional histoculture and the MTT end point. Int J Cancer 51: 489-498, 1992.

36. Suto A, Kubota T, Shimoyama Y, Ishibiki K and Abe O: MTT assay with reference to the clinical effect of chemotherapy. J Surg Oncol 42: 28-32, 1989.

37. Furukawa T, Kubota T, Suto A, et al: Clinical usefulness of chemosensitivity testing using the MTT assay. J Surg Oncol 48: 188-193, 1991.

38. Miller BE, Miller FR and Heppner GH: Assessing tumor drug sensitivity by a new in vitro assay which preserves tumor heterogeneity and subpopulation interactions. J Cell Physiol 3 (Suppl): 105-116, 1984.

39. Takamura Y, Kobayashi H, Taguchi T, Motomura K, Inaji H and Noguchi S: Prediction of chemotherapeutic response by collagen gel droplet embedded culture-drug sensitivity test in human breast cancers. Int J Cancer 98: 450-455, 2002.
40. Nagai N, Minamikawa K, Mukai K, Hirata E, Komatsu M and Kobayashi H: Predicting the chemosensitivity of ovarian and uterine cancers with the collagen gel droplet culture drugsensitivity test. Anticancer Drugs 16: 525-531, 2005.

41. Vescio RA, Connors KM, Kubota T and Hoffman RM: Correlation of histology and drug response of human tumors grown in native-state three-dimensional histoculture and in nude mice. Proc Natl Acad Sci USA 88: 5163-5166, 1991.

42. Ezdinli EZ, Gelber R, Desai DV, Falkson G, Moertel CG and Hahn RG: Chemotherapy of advanced esophageal carcinoma: Eastern Cooperative Oncology Group experience. Cancer 46: 2149-2153, 1980

43. Engstrom PF, Lavin PT and Klaassen DJ: Phase II evaluation of mitomycin and cisplatin in advanced esophageal carcinoma. Cancer Treat Rep 67: 713-715, 1983.

44. Ajani JA, Ilson DH, Daugherty K, Pazdur R, Lynch PM and Kelsen DP: Activity of taxol in patients with squamous cell carcinoma and adenocarcinoma of the esophagus. J Natl Cancer Inst 86: 1086-1091, 1994.

45. Sandler AB, Kindler HL, Einhorn LH, et al: Phase II trial of gemcitabine in patients with previously untreated metastatic cancer of the esophagus or gastroesophageal junction. Ann Oncol 11: 1161-1164, 2000.

46. Kubota T, Sasano N, Abe O, et al: Potential of the histoculture drug-response assay to contribute to cancer patient survival. Clin Cancer Res 1: 1537-1543, 1995.

47. Abe S, Kubota T, Matsuzaki SW, et al: Chemosensitivity test is useful in evaluating the appropriate adjuvant cancer chemotherapy for stage III non-scirrhous and scirrhous gastric cancers. Anticancer Res 19: 4581-4586, 1999. 